\title{
Local and Global Panoramic Imaging for Fluorescence Bladder Endoscopy
}

\author{
Behrens, Alexander and Stehle, Thomas and Gross, Sebastian and \\ Aach, Til \\ Institute of Imaging and Computer Vision \\ RWTH Aachen University, 52056 Aachen, Germany \\ tel: +49 24180 27860, fax: +49 2418022200 \\ web: www.lfb.rwth-aachen.de
}

in: Engineering in Medicine and Biology Society, EMBC 2009. 31th Annu. Int. Conf. of the IEEE.

See also BiBT $\mathrm{E}_{\mathrm{E}} \mathrm{X}$ entry below.

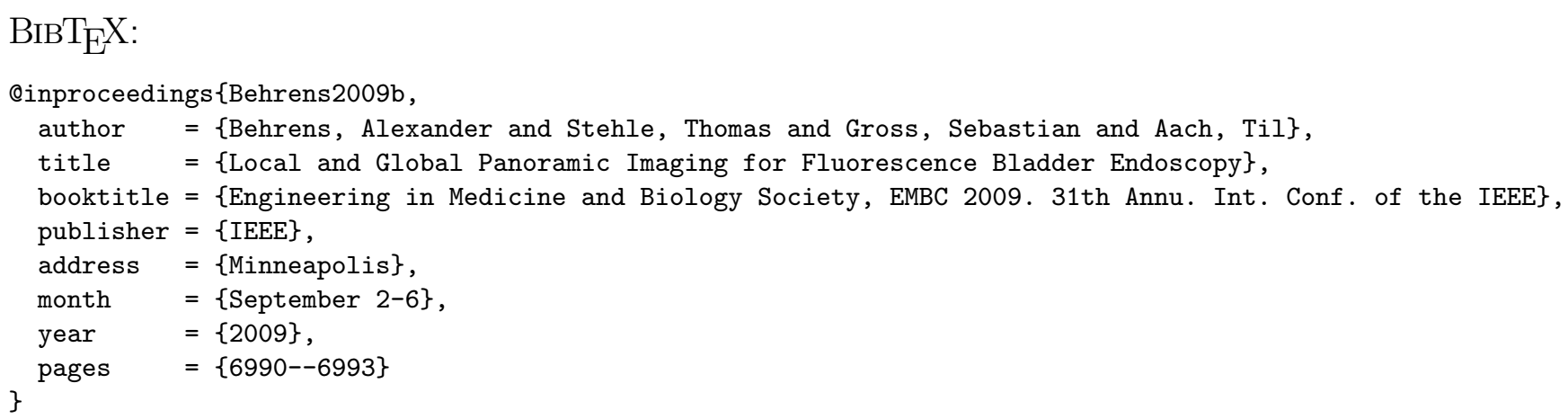

(C) 2009 IEEE. Personal use of this material is permitted. However, permission to reprint/republish this material for advertising or promotional purposes or for creating new collective works for resale or redistribution to servers or lists, or to reuse any copyrighted component of this work in other works must be obtained from the IEEE. 


\title{
Local and Global Panoramic Imaging for Fluorescence Bladder Endoscopy
}

\author{
Alexander Behrens, Thomas Stehle, Sebastian Gross, and Til Aach \\ Institute of Imaging \& Computer Vision, RWTH Aachen University, 52056 Aachen, Germany
}

\begin{abstract}
Endoscopic treatment of bladder cancer is more and more often based on photodynamic diagnostics (PDD), a specialized endoscopic technique where a narrow-band bluish illumination causes tumors to fluoresce reddish. Contrast between tumors and healthy bladder tissue is thus noticeably increased compared to white light endoscopy. A downside of PDD is the low illumination power, which requires that the distance between endoscope and bladder wall be kept low, thus resulting in a small field of view (FOV). We therefore describe an approach to combine several successive frames into a local PDD panorama, which provides a larger and sufficiently bright FOV for treatment. Furthermore, the endoscopic cancer treatment generally starts with a complete scan of the bladder to detect the tumors. For diagnosis, navigation and reporting, a global overview image of the bladder wall is often desired. While construction of such a global panorama can be based on the same algorithm as the local panorama, direct planar visualization of the sphere-shaped bladder may cause severe distortions. Apart from the global panorama computation itself, we therefore analyze these distortions, and provide an alternative visualization which is based on bladder depictions used in standard reporting forms and anatomy textbooks.
\end{abstract}

\section{INTRODUCTION}

Medical diagnosis and therapy of urinary bladder cancer are usually carried out during a cystoscopy, in which an endoscope is introduced through the urethra into the bladder. Malignant tissues of the bladder wall can then be removed with the use of endoscopic tools. Since bladder cancer is the fourth most common malignancy among males in industrial countries, with over 68,000 new cases in 2008 in the USA alone [1], this procedure is a regular intervention.

Under white illumination, the structure of small and flat tumors does not differ strongly from the surrounding tissue. Thus, the risk of missing tumors during therapy is high. To enhance the visual contrast between malignant and benign tissue, so-called photodynamic diagnostics (PDD) endoscopy applied by a PDD imaging platform like Olympus EVIS EXERA II can be used, where the illumination extends only over a bluish narrow band between $380 \mathrm{~nm}$ and $450 \mathrm{~nm}$. Activated by 5 -aminolevulinic acid or Hexvix ${ }^{\circledR}$, tumors then fluoresce reddish, thus resulting in a visual improved tissue contrast as illustrated in Fig. 1 (upper row). Due to the low illumination power, the distance between endoscope and bladder wall must be kept small to obtain sufficiently exposed images. Otherwise the PDD images lack in structure, contrast and illumination, as shown in Fig. 1 (lower left). Thus, the fluorescence images generally provide a small field of view

We thank Olympus Winter \& Ibe GmbH, Hamburg, Germany for funding this project.

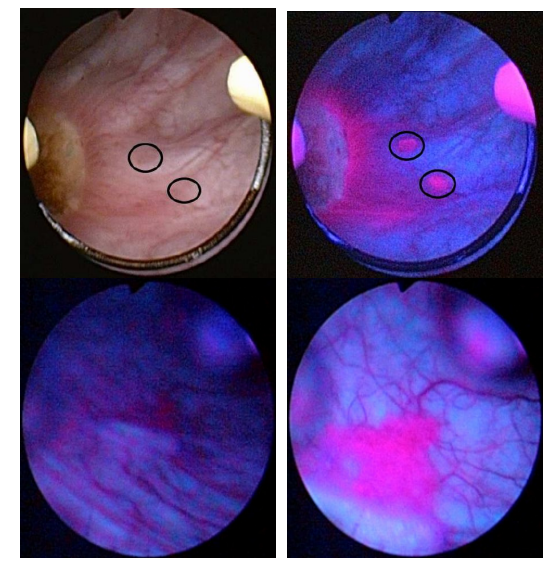

Fig. 1. Papillary tumors under white (upper left) and PDD (upper right) illumination. PDD images taken with a large (lower left) and small distance (lower right) between endoscope and bladder wall.

(FOV), which impedes navigation and relocating multifocal tumors.

To provide a larger and sufficiently bright FOV of the operating field at a time, we describe an approach to compute a local panorama from a short sequence of PDD images taken from slightly different perspectives. While mosaicking algorithms based on whitefield endoscopy exist [2]-[4], we developed an algorithm specifically tailored to PDD [5].

The cancer treatment generally starts and ends with a complete scan of the bladder to detect all tumors, to analyze the spatial distribution, which can improve diagnosis, and to make sure that all tumors were removed. Clinical studies showed that multifocality of carcinoma in situ (CIS) of the bladder increases the probability of invasive urothelial carcinoma, and reduces the survival rate of the patient [6]. Thus, a global overview image of the bladder is often desired for diagnosis, navigation as well as for post-interventional documentation. The construction of such a global PDDpanorama can be based on the same algorithm as the local panorama, described in section II. However, the wide solid angle covered by a full bladder scan in connection with the sphere-like bladder shape causes severe distortions in the panorama image. The reason for this is that, during the mosaicking process, all images are projected onto the plane of the same reference image, which results in increasing geometric distortions of images acquired later on. Furthermore these distortions prevent a planar visualization of the panorama, and may interrupt the panorama computation. While previous approaches [2]-[4] have not addressed these limitations, we discuss the systematic distortions in 
section III. In section IV, we then describe an alternative to global panorama computation of the bladder, where the bladder is approximated by a hemicube. Its planar faces are covered by solid angles, which are sufficiently low to allow planar mosaicking and visualization without excessive distortions. Moreover, the hemicube can be unfolded, resulting in a bladder depiction similar to bladder representations in evidence protocols and anatomy textbooks. Conclusions are drawn in section $\mathrm{V}$.

\section{IMAGE MOSAICKING ALGORITHM}

For the composition of a local PDD panorama an iterative mosaicking algorithm is applied. First, the input images of endoscopic PDD video sequence are subsampled and rectified from the lens distortion based on camera calibration data [7]. Then the relevant ellipse-shaped image region (see Fig. 1) is extracted. A typical property of PDD endoscopy images is that they exhibit reasonable vasculature contrast over almost the entire bladder wall. We therefore perform a feature-based registration. To account for the scale variations of vasculature (cf. [8]), distinctive scale invariant keypoints are extracted from gray value images by the Scale Invariant Feature Transform (SIFT) [9]. The resulting high dimensional feature descriptors of an image pair are matched using the minimum Euclidean distance measurement. Based on the resulting point correspondences an image transformation, a so-called 2D-homography, is estimated. Since the video sequence describes a non-rigid camera movement and projective errors of two sequential images can be neglected, the homography is sufficiently determined by an affine transformation model. It provides six degrees of freedom, parameterized by a translation vector $\vec{t}^{T}=\left(t_{x}, t_{y}\right)$, rotation angle $\alpha$, scales $s_{x}$ and $s_{y}$, and skew $a$. In homogeneous coordinates the homography matrix $\mathbf{H}$ can be written as

$$
\mathbf{H}=\left[\begin{array}{lll}
a & b & c \\
d & e & f \\
0 & 0 & 1
\end{array}\right]=\left[\begin{array}{cc}
\mathbf{A} & \vec{t} \\
\overrightarrow{0}^{T} & 1
\end{array}\right]
$$

with

$$
\mathbf{A}=\left[\begin{array}{ll}
1 & a \\
0 & 1
\end{array}\right]\left[\begin{array}{cc}
s_{x} & 0 \\
0 & s_{y}
\end{array}\right]\left[\begin{array}{rr}
\cos (\alpha) & -\sin (\alpha) \\
\sin (\alpha) & \cos (\alpha)
\end{array}\right]
$$

To ensure a robust homography estimation, unavoidable false point correspondences are rejected by a modified RANSAC (RANdom SAmple Consensus) model fitting algorithm [10]. Finally, a blending method based on a linear mutual weight distribution is applied to remove visual artifacts. The subsequent images are then warped to the coordinate system of the reference image, e.g. the first image of the sequence, until the local panorama is complete. Additional and more detailed descriptions of the algorithm are given in [5]. A local panorama computed from 580 frames PDD endoscopic video sequence is shown in Fig. 2.

\section{MAP PROJECTION}

While the local panorama in Fig. 2 exhibits only minor distortions, a flat global panorama for a larger FOV can not

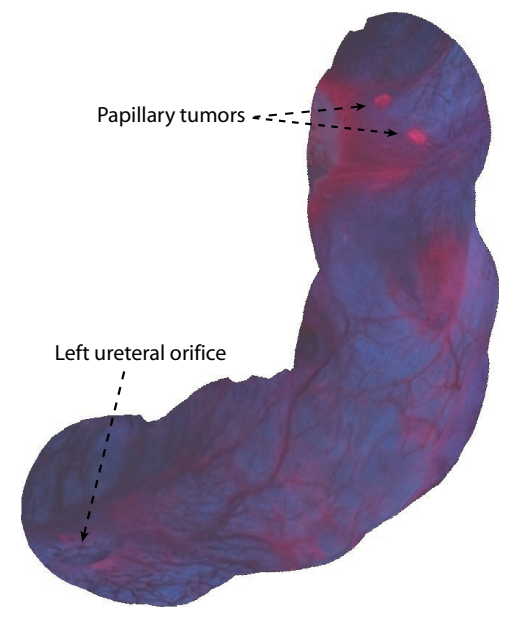

Fig. 2. PDD panoramic image computed from a video sequence of 580 frames, showing two papillary tumors on the upper left wall relative to the left ureteral orifice.

be calculated without excessively stretching pixels [11]. In general the distortion becomes visually disturbing once the FOV exceeds about $90^{\circ}$. This thus affects the composition of a global panoramic image showing the whole internal bladder surface, as illustrated in Fig. 3. Although the distortions

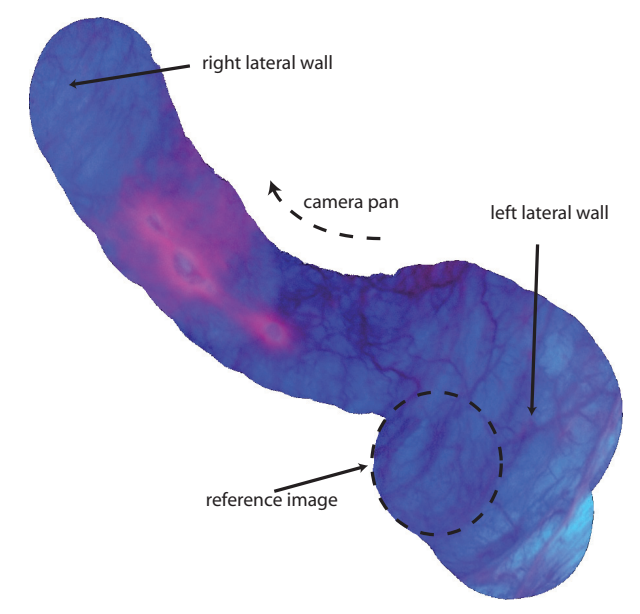

Fig. 3. Local panorama showing major distortions in the upper left corner, caused by a horizontal panning of the cystoscope from the left to the right lateral bladder wall.

of the panorama in Fig. 3 around the reference image are small, they become worse for larger FOVs. They result from a horizontal panning of the cystoscope from the left to the right lateral bladder wall. After a reference coordinate system is set by, e.g. the first recorded image, image $i$ of the sequence is warped by the global transformation matrix $\mathbf{H}_{i}^{g}$. Consequently the next image $i+1$ will be transformed by

$$
\begin{aligned}
\mathbf{H}_{i+1}^{g} & =\mathbf{H}_{i, i+1} \cdot \mathbf{H}_{i}^{g}=\mathbf{H}_{i, i+1} \cdot \ldots \cdot \mathbf{H}_{2,3} \cdot \mathbf{H}_{1,2} \\
& =\prod_{k=0}^{i-1} \mathbf{H}_{i-k, i-k+1}
\end{aligned}
$$

with the local homography $\mathbf{H}_{i, i+1}$ between image $i$ and $i+1$. Although the transformation parameters between two subsequent images of the curved bladder surface can be small, the 
visual distortion between image $n$ and the reference image increases continuously according to eq. (3).

From a geometric point of view this effect can be described considering the projection of a single circle of latitude of a given sphere onto a plane, as shown in Fig. 4. This mapping can be illustrated by cutting the latitudes into

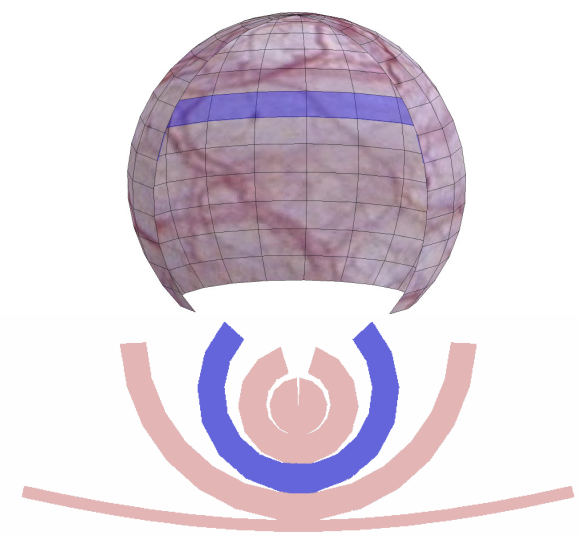

Fig. 4. Systematic distortions caused by the planar projection of individual circles of latitudes of a hemisphere.

stripes and unfolding them onto a plane. They result then in circle-like segments. This characteristic can also be found in Fig. 3 and is unavoidable since the upper and lower edge of the latitude stripe differ in length.

To reduce this effect cylindrical or spherical projections [12] are usually applied for global panoramas. Due to the fact that any map projection produces distortions of the object surface in some fashion, as proved by Gauss's Theorema Egregium [13], they can be categorized according to the quantity they preserve: Angle (conformal), distance (equidistant), and area (equal-area) projections. For bladder cancer treatment the latter type is most important, since the correct size and area of the cancer tumors are essential for diagnosis and therapy.

\section{HEMICUBE MAP}

Although the bladder could be adequately approximated by a $3 \mathrm{D}$ sphere-shape, a practical representation is restricted by clinical requirements to a planar map projection. Image compositions can thus be easily printed, visualized and exchanged in common existing medical picture archiving and communication systems (PACS).

To cope with these limitations, and motivated by common anatomical bladder depictions, we approximate the urinary bladder by five tangential planes, each representing a map projection onto a hemicube face. (Since the front wall is usually examined by deforming the bladder instead of using a retrospective optics, an entire cube model including all six planes is not applicable.) Thus, the mapping onto the hemicube planes can be performed by five independent planar projections, respectively five local panoramas. Each represents one of the anatomical bladder regions (back wall, left and right lateral wall, upper wall, ostium), defined by common urological depictions, illustrated in Fig. 5.
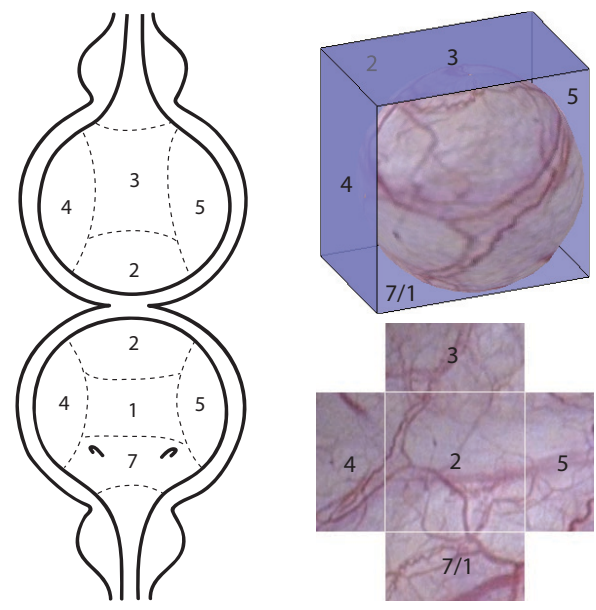

Fig. 5. Urological depiction with labeled bladder regions (left), related to faces of the hemicube bladder approximation (upper right). Emulated overview of the bladder surface by unfolding the hemicube (lower right).

Furthermore a global panorama can be emulated by unfolding all hemicube planes and displaying the local panoramas side by side.

The projection of a single patch of the bladder surface onto a hemicube plane is illustrated in Fig. 6. Considering

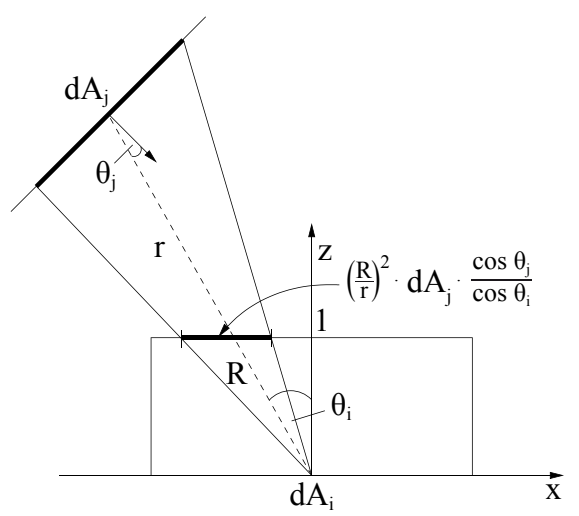

Fig. 6. Projection of an differential patch onto the unit hemicube.

an orthonormal coordinate system in Euclidean domain with its origin $O$ in the center of the hemicube, a differential patch of the bladder surface $d A_{j}$ is projected in the plane of $d A_{i}$ of the unit hemicube. The projected patch is then determined by

$$
T\left(d A_{j}\right)=W_{i j} \cdot\left(\frac{R}{r}\right)^{2} \cdot d A_{j} \cdot \frac{\cos \theta_{j}}{\cos \theta_{i}},
$$

with the indicator variable

$$
W_{i j}=\left\{\begin{array}{lll}
1 & : & d A_{j} \text { is visible from } d A_{i} \\
0 & : & \text { otherwise }
\end{array} .\right.
$$

With the relation $R=\frac{1}{\cos \theta_{i}}$, eq. (4) becomes

$$
\begin{aligned}
T\left(d A_{j}\right) & =W_{i j} \cdot \frac{1}{r^{2}} \cdot \frac{1}{\cos ^{2} \theta_{i}} \cdot d A_{j} \cdot \frac{\cos \theta_{j}}{\cos \theta_{i}} \\
& =W_{i j} \cdot \underbrace{\frac{\cos \theta_{j} \cos \theta_{i}}{\pi r^{2}} d A_{j}}_{\Delta F} \cdot \frac{\pi}{\cos ^{4} \theta_{i}} .
\end{aligned}
$$

Equivalent to radiosity methods [14] eq. (6) contains the indicator variable, the form factor $\Delta F$, which specifies the 
fraction of the energy leaving one surface which lands on another, and a term describing the hemicube geometry. If the patches $d A_{j}$ and $d A_{i}$ are considered as planes, the transformation $T\left(d A_{j}\right)$ can also be described by a homography matrix, as stated in eqs. (1) and (3).

Thus, the algorithm results in the following: First, one hemicube plane is selected by the physician and the corresponding bladder region (Fig. 5) is scanned with the endoscope. Assuming a continuous bladder scan, the first image is taken as reference. Then the subsequent images are warped into the reference coordinate system using the calculated homographies, and the local panorama is successively composed. Since no orientation of the viewing direction is measured by the endoscope system, the indicator variable (eq. (5)) has to be set explicitly by stopping the image recording when the FOV of the endoscope enters a new bladder region. In an iterative process the next hemicube plane is selected until all local panoramas are constructed.

Due to the limited panoramas' FOVs, the visual distortions are small. Furthermore the applied homographies transform all images to the scale of the reference image. As long as projective errors can be neglected, an equal-area mapping is performed. Finally, the hemicube planes are unfolded and the local panoramas are displayed side by side on a monitor. Using a visualization tool the physician can select each panorama for a closer view, and the related bladder region is then highlighted in an urological bladder model, as illustrated in Fig. 7. Thus, panoramic image compositions of local bladder regions can be easily visualized, documented and exchanged over PACS. Furthermore the hemicube visualization can provide information about spatial tumor distribution, and assist in navigation, like re-locating tumors.

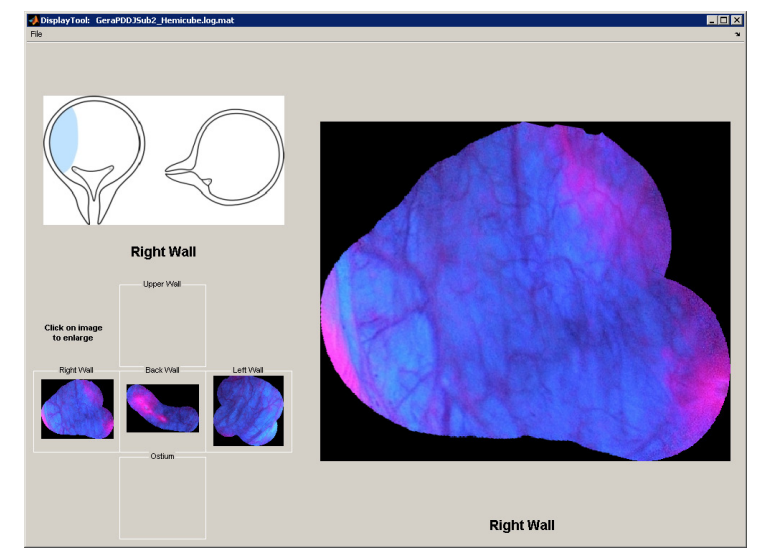

Fig. 7. Visualization tool of local panoramas of hemicube planes, arranged in an unfolded hemicube structure (lower left). The selected local panorama (right) is highlighted in the urological bladder depiction (upper left).

\section{Conclusions}

We have discussed both local and global panorama computation in PDD-endoscopy of the bladder. In both cases, panorama calculation is based on a mosaicking algorithm for PDD-endoscopic data, which exploits the vasculature of the bladder wall. While for a sufficiently low solid angle, the images of the PDD sequence can be directly assembled into a local panoramic image, computing a global bladder overview fails because of the excessive geometric distortions involved. The algorithms in [2], [3] works on explanted, flattened bladders, and do thus not address this problem. We discussed and analyzed quantitatively how these systematic distortions are caused, why they become even worse for larger FOVs, and finally result in matching interruptions. Starting from urological bladder depictions commonly used in clinical routine, we overcome the above dilemma by a piecewise planar approximation of the sphere-shaped bladder, leading to a hemicube representation. The projection onto each planar face of the hemicube has been discussed, and can be treated as a local panorama problem. A global panorama is then obtained by unfolding the hemicube faces, controlled by a visualization tool. By selecting the different panoramas the related bladder regions are highlighted in a common urological bladder model, providing an intuitive usability. Although further features like the automatic detection and labeling of overlapping image structures along two adjacent hemicube planes or the automatic image assignment controlled by additional orientation information can increase the usability, preliminary feedback of surgeons showed already high potential for surgery planning and guided re-locating of malignant tissue. Further objective and statistical clinical evaluations will be carried out in future work.

\section{REFERENCES}

[1] American Cancer Society, "Cancer facts and figures 2008."

[2] R. Miranda-Luna, C. Daul, W. Blondel, Y. Hernandez-Mier, D. Wolf, and F. Guillemin, "Mosaicing of bladder endoscopic image sequences: Distortion calibration and registration algorithm," IEEE Trans. Biomed. Eng., vol. 55, no. 2, pp. 541-553, Feb 2008.

[3] Y. Hernandez-Mier, W. Blondel, C. Daul, D. Wolf, and G. BourgHeckly, "2-d panoramas from cystoscopic image sequences and potential application to fluorescence imaging," in Modeling and Control in Biomedical Systems, vol. 6, no. 1. Elsevier, Sep 2006.

[4] S. Seshamani, W. Lau, and G. Hager, "Real-time endoscopic mosaicking," in MICCAI, ser. LNCS, vol. 1, no. 1. Springer, 2006, pp. 355363.

[5] A. Behrens, "Creating panoramic images for bladder fluorescence endoscopy," Acta Polytechnica Journal of Advanced Engineering, vol. 48, no. 3, pp. 50-54, June 2008.

[6] R. E. Orozco, A. A. Martin, and W. M. Murphy, "Carcinoma in situ of the urinary bladder. clues to host involvement in human carcinogenesis." Cancer, vol. 74, no. 1, pp. 115-122, Jul 1994.

[7] T. Stehle, D. Truhn, T. Aach, C. Trautwein, and J. Tischendorf, "Camera calibration for fish-eye lenses in endoscopy with an application to 3D reconstruction," in IEEE ISBI, 2007, pp. 1176-1179.

[8] T. Aach, C. Mayntz, P. Rongen, G. Schmitz, and H. Stegehuis, "Spatiotemporal multiscale vessel enhancement for coronary angiograms," in Medical Imaging 2002, vol. 4684, pp. 1010-1021.

[9] D. Lowe, "Distinctive image features from scale-invariant keypoints," in $I J C V$, vol. 60, no. 2, Nov 2004, pp. 91-110.

[10] M. A. Fischler and R. C. Bolles, "Random sample consensus: A paradigm for model fitting with applications to image analysis and automated cartography," Commun. ACM, vol. 24, no. 6, pp. 381-395, June 1981.

[11] R. Szeliski, "Image alignment and stitching: A tutorial," Microsoft Research, Tech. Rep. MSR-TR-2004-92, 2006.

[12] R. Szeliski and H.-Y. Shum, "Creating full view panoramic image mosaics and environment maps," in SIGGRAPH, 1997, pp. 251-258.

[13] K. F. Gauss, "General investigations of curved surfaces of 1827 and 1825," The Princeton University Library, 1902.

[14] M. F. Cohen and D. P. Greenberg, "The hemi-cube: a radiosity solution for complex environments," in SIGGRAPH, 1985, pp. 31-40. 